\title{
Effects of Structural Modification on Second Harmonic Generation in Collagen
}

P. C. Stoller, K. M. Reiser, P. M. Celliers, A. M. Rubenchik

This article was submitted to

SPIE Conference on Visualization and Data Analysis, San Jose, CA, January 20-25, 2003

U.S. Department of Energy

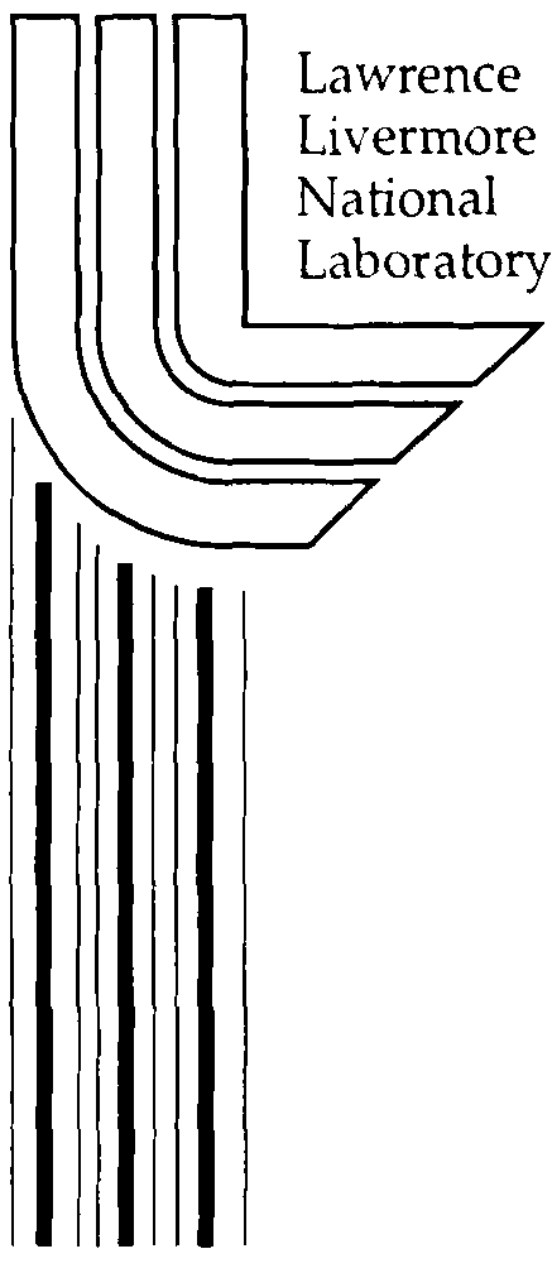

April 4, 2003 


\section{DISCLAIMER}

This document was prepared as an account of work sponsored by an agency of the United States Government. Neither the United States Government nor the University of California nor any of their employees, makes any warranty, express or implied, or assumes any legal liability or responsibility for the accuracy, completeness, or usefulness of any information, apparatus, product, or process disclosed, or represents that its use would not infringe privately owned rights. Reference herein to any specific commercial product, process, or service by trade name, trademark, manufacturer, or otherwise, does not necessarily constitute or imply its endorsement, recommendation, or favoring by the United States Government or the University of California. The views and opinions of authors expressed herein do not necessarily state or reflect those of the United States Government or the University of California, and shall not be used for advertising or product endorsement purposes.

This is a preprint of a paper intended for publication in a journal or proceedings. Since changes may be made before publication, this preprint is made available with the understanding that it will not be cited or reproduced without the permission of the author.

This work was performed under the auspices of the United States Department of Energy by the University of California, Lawrence Livermore National Laboratory under contract No. W-7405-Eng-48.

This report has been reproduced directly from the best available copy.

Available electronically at http://www.doc.gov/bridge

Available for a processing fee to U.S. Department of Energy

And its contractors in paper from

U.S. Department of Energy

Office of Scientific and Technical Information

P.O. Box 62

Oak Ridge, TN 37831-0062

Telephone: (865) 576-8401

Facsimile: (865) 576-5728

E-mail: reports@adonis.osti.gov

Available for the sale to the public from

U.S. Department of Commerce

National Technical Information Service

5285 Port Royal Road

Springfield, VA 22161

Telephone: (800) 553-6847

Facsimile: (703) 605-6900

E-mail: orders@ntis.fedworld.gov

Online ordering: http://www.ntis.gov/ordering.htm

OR

Lawrence Livermore National Laboratory

Technical Information Department's Digital Library

http://www.llnl.gov/tid/Library.html 


\title{
Effect of structural modification on second harmonic generation in collagen
}

\author{
Patrick C. Stoller ${ }^{*}$, Karen M. Reiser ${ }^{2}$, Peter M. Celliers ${ }^{1}$, and Alexander M. Rubenchik ${ }^{3}$ \\ ${ }^{1}$ M Div., Lawrence Livermore Natl. Lab, L-174, P.O. Box 808, Livermore, CA, USA 94551 \\ ${ }^{2}$ Dept. of Neuro. Surg., Univ. of California, Davis, CA, 1 Shields Ave., Davis, CA, USA 95616 \\ ${ }^{3}$ NIF Prog., Lawrence Livermore Natl. Lab, L-399, P.O. Box 808, Livermore, CA, USA 94551
}

\begin{abstract}
The effects of structural perturbation on second harmonic generation in collagen were investigated. Type I collagen fascicles obtained from rat tails were structurally modified by increasing nonenzymatic cross-linking, by thermal denaturation, by collagenase digestion, or by dehydration. Changes in polarization dependence were observed in the dehydrated samples. Surprisingly, no changes in polarization dependence were observed in highly crosslinked samples, despite significant alterations in packing structure. Complete thermal denaturation and collagenase digestion produced samples with no detectable second harmonic signal. Prior to loss of signal, no change in polarization dependence was observed in partially heated or digested collagen.
\end{abstract}

Keywords: Second harmonic generation, collagen, polarization, rat tail tendon, non-enzymatic glycation

\section{INTRODUCTION}

Collagen, the major structural protein in the body, has long been observed to have significant second order nonlinear susceptibility. With the advent of ultrashort pulse lasers, it has become possible to study second harmonic generation (SHG) in collagen at high resolution without damaging the tissue ${ }^{1 \cdot 13}$.

Fibrillar collagen molecules form highly organized structures, comprising long fibrils with diameters of $15 \mathrm{~nm}$ to 500 $\mathrm{nm}$, that provide structural support in organisms ${ }^{14}$. Collagen has an exceptionally long half-life, measured in decades. As a consequence it undergoes constant post-translational modification through genetically controlled processes, such as lysyl oxidase-mediated crosslinking ${ }^{15-16}$, and stochastic processes, such as nonenzymatic glycation and oxidation ${ }^{17-18}$. These modifications are responsible for many of the degenerative changes associated with aging. In some cases, abnormalities in specific post-translational modifications are associated with disease states.

SHG microscopy provides high resolution $(\sim 1 \mu \mathrm{m})$ images of collagen structure as a function of depth in tissue, to a depth of a few hundred micrometers. In previous studies ${ }^{10-11}$, we observed a high degree of variability (two to three orders of magnitude) in the strength of the second harmonic signal in two-dimensional scans of collagenous tissues, even in structurally homogeneous tissues such as rat tail tendon. This variability makes it difficult to use the strength of the second harmonic signal to assess changes in collagen structure. Possibly, if enough data points were collected in control samples and structurally modified samples, a statistically significant difference in signal strength could be observed. However, one of the important advantages of SHG microscopy-high resolution-would be lost. Furthermore, data collection would probably be too time consuming for clinical application.

We also observed that the polarization dependence of the SHG signal fluctuated less than the intensity as a function of position ${ }^{10-11}$. This led us to ask: Do biologically relevant modifications of collagen structure result in changes in the polarization dependence of SHG that can be readily detected? To answer this question, we studied the polarizationdependence of SHG in collagen that had been structurally altered in ways that simulated naturally occurring derangements. Type I collagen fibrils were subjected to thermal denaturation, collagenase digestion, non-enzymatic cross-linking, and dehydration. We compared the polarization dependence in these samples with that observed in control samples.

"stoller2@llnl.gov; phone 1-925-423-2466; fax 1-925-424-2778; www.llnl.gov 


\section{THEORY}

\section{1 Measuring the polarization dependence of the SHG signal in collagen}

The dependence of SHG in collagen on the orientation of the linear input polarization has been successfully modeled assuming a second order nonlinear susceptibility tensor with cylindrical $C_{*}$, symmetry ${ }^{11.20}$. Because of cylindrical symmetry and other symmetries resulting from the fact that the wavelengths used are far from collagen electronic resonances, a single parameter describes the polarization dependence. We present a brief summary of this model and the method that we use to measure this parameter.

The nonlinear susceptibility tensor for cylindrically symmetric materials can be written as

$$
d_{i j k}^{(2)}=a s_{i} s_{j} s_{k}+b\left(s_{i} \delta_{j k}+s_{j} \delta_{i k}+s_{k} \delta_{i j}\right)
$$

where $\bar{s}$ is a unit vector along the fibril axis and a and $\mathrm{b}$ are independent parameters of the nonlinear susceptibility tensor; we define $\gamma=b / a$. Figure 2 illustrates the orientation of the collagen fibril, laser propagation direction, and laser polarization direction with respect to each other. Making the paraxial approximation, the polarization dependence of the SHG signal is then given by,

$$
I_{S H G} \propto \frac{1}{8}\left(3+20 \gamma+40 \gamma^{2}\right)-\frac{1}{2}\left(1+6 \gamma+8 \gamma^{2}\right) \operatorname{Cos}(2 \alpha+2 \phi)+\frac{1}{8}(1+4 \gamma) \operatorname{Cos}(4 \alpha+4 \phi),
$$

where $\alpha$ is the angle that the laser polarization makes with respect to the $\mathrm{x}$-axis and $\phi$ is the angle that the collagen fibril makes with respect to the y-axis. We use a polarization-modulation technique (see Experiment) that continuously rotates the linear polarization of the input light from 0 to 180 degrees at a frequency $\Omega$ in a saw-tooth pattern, so that $\alpha=\pi \Omega$. Substituting into Eq. 2, we have

$$
I_{S H G} \propto \frac{1}{8}\left(3+20 \gamma+40 \gamma^{2}\right)-\frac{1}{2}\left(1+6 \gamma+8 \gamma^{2}\right) \operatorname{Cos}(2 \pi \Omega t+2 \phi)+\frac{1}{8}(1+4 \gamma) \operatorname{Cos}(4 \pi \Omega t+4 \phi) .
$$

Thus, if we measure the component of the second harmonic light modulated at frequency $\Omega$ (the first modulation harmonic, $\left.I_{F M H}\right)$, we obtain a signal proportional to $-\frac{1}{2}\left(1+6 \gamma+8 \gamma^{2}\right)$ while if we measure the component at $2 \Omega$ (the second modulation harmonic, $I_{S M H}$, we obtain a signal proportional to $\frac{1}{8}(1+4 \gamma)$. From the ratio $R=-\frac{1}{4+8 \gamma}$ of these two signals, we can calculate $\gamma$. Using Eq. 1 and $\gamma=b / a$ and defining a new coordinate system where $X$ corresponds to the collagen fibril axis and $Y$ to the direction normal to both the fibril and the laser beam, it is possible to obtain $\frac{d_{X Y Y}}{d_{X X X}}=\frac{\gamma}{3 \gamma+1}$. Using $R=-\frac{1}{4+8 \gamma}$ we can also write, $\frac{d_{X Y Y}}{d_{X X X}}=\frac{1+4 R}{3+4 R}$. Thus, polarization-modulation allows direct measurement of the ratio between the two independent elements in the second order nonlinear susceptibility tensor.

By chopping the incident laser beam using an optical chopper, we can also measure the polarization-independent component of the signal. We will use $S$ to denote the ratio of the signal at the first modulation harmonic of the electrooptic modulator to the signal at the optical chopper frequency $S$. We should note that $S$ does not correspond exactly to the ratio of the first two terms in Eq. 3 above. The optical chopper produces a modulation that includes a DC component as well as components at the first and higher harmonics of the chopper frequency. If we rewrite Eq. 3 to include the 
effect of chopping the beam and we let $\Omega_{\text {chop }}$ be the chopper frequency, $C_{D C}$ the DC component of the chopped beam, and $C_{\Omega_{\text {chop }}}$ the component at the chopper frequency, we have (leaving out terms at frequencies other than $\Omega_{\text {chop }}$ and $\Omega$ )

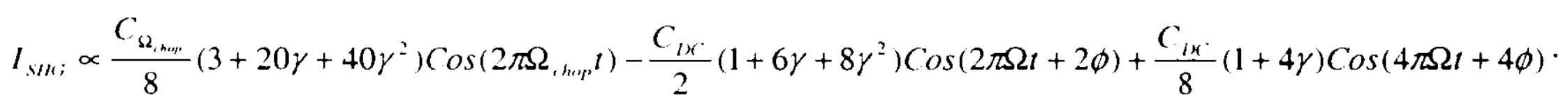

Thus, $S$ must be multiplied by a correction factor $C_{\Omega_{\text {chop }}} / C_{D C}$ to obtain the correct value for the ratio of the first two terms in Eq. 3.

The steps leading from Eq. 1 to Eq. 3 are discussed in detail elsewhere ${ }^{11}$. In deriving Eq. 3 we have assumed normal incidence on the fibrils (incident beam normal to the fibril axis) and omitted linear optical effects that could affect the polarization dependence-birefringence and polarization-dependent scattering. We have also assumed that all of the fibrils in the interaction region (the region around the focus of the beam where the intensity is large enough to contribute significantly to $\mathrm{SHG}$ ) are oriented in the same direction. We have shown in previous work ${ }^{11}$ that conditions under which these assumptions are valid can be attained by using thin, frozen sections of highly uniform rat tail tendon tissue. We have also made the paraxial approximation, neglecting the fact that focusing through a high numerical aperture microscope objective can modify the polarization of the beam.

\subsection{Quartz calibration}

To independently confirm the accuracy of the polarization-modulation technique for measuring $R$ and $S$ in collagen, we applied it to a well-characterized nonlinear crystal. We chose a quartz waveplate-a crystal cut where the optical axis lies in the plane of the waveplate, normal to the beam propagation direction. The second-order nonlinear susceptibility tensor in quartz (choosing the $\mathrm{Z}$-axis as the optic axis) is given by

$$
d_{X X X}=-d_{X Y Y}=-d_{Z X Y}=-d_{Z Y X},
$$

where $d_{X X X}=0.40 \mathrm{pm} / \mathrm{V}^{21}$ and all of the other tensor elements are zero. The surface of the waveplate lies in the $X-Z$ plane; the laser propagates in the Y-direction. Using Eq. 5, we obtain an expression for the second harmonic signal generated by the quartz plate as a function of polarization angle $\alpha$ (measured from the $\mathrm{X}$-axis),

$$
I_{S H G} \propto \frac{3}{8}+\frac{1}{2} \operatorname{Cos}(2 \pi \alpha)+\frac{1}{8} \operatorname{Cos}(4 \pi \alpha)
$$

From Eq. 6 , it is clear that the ratio $R$ between the signal at the second modulation harmonic and the signal at the first modulation harmonic should be 0.25 and the ratio $S_{\text {corr }}$ between the signal at the first modulation harmonic and the DC component of the signal (after correction for the effect of the chopper modulation itself, see above) should be $4 / 3$. The value of the correction factor is calculated by assuming a certain beam profile for the laser beam and determining how the chopper modulates such a beam. Since we must chop the beam before it goes through the spatial filter and beam expander-otherwise the beam size exceeds the size of the chopper blades-the beam is elliptical, making the calculation complex. We choose instead to use one of the measurements in quartz to calibrate the correction factor (see Calibration).

\section{EXPERIMENT}

\subsection{Optical setup}

The optical setup used for these studies has been previously described" ; Figure 1 provides a diagram of the setup. Briefly, we used a Ti:Sapphire oscillator (Mira; Coherent Inc., Santa Clara, CA) to generate linearly polarized 200 fs pulses at a wavelength of $800 \mathrm{~nm}$ and with a maximum energy of $5 \mathrm{~nJ}$ and at a repetition rate of $76 \mathrm{MHz}$. A Faraday isolator was used to prevent back-scattered light from entering the laser cavity and interfering with mode-locking. The beam passed through a half-wave plate followed by a polarizing beam-splitter; the half-wave plate was rotated to control the power incident on the sample. The p-polarized light transmitted through the beam-splitter was chopped at $1.5 \mathrm{kHz}$ 
using an optical chopper and then passed through an electro-optic modulator (360-80; Conoptics, Danbury, CT) with its axes oriented at 45 degrees to the polarization of the light. The beam was passed through a spatial filter and beam expander (a $50 \mathrm{~mm}$ lens focused the beam onto a $50 \mu \mathrm{m}$ pinhole). The beam then passed through a quarter-wave plate with its axes oriented at 45 degrees to those of the electro-optic modulator.

A Mitutoyo 20X ( $\mathrm{NA}=0.42$ ) microscope objective was used to focus the beam onto the sample. An iris was used to allow only the central maximum of the Airy pattern produced by the spatial filter to enter the back-aperture of the microscope objective. The transmitted second harmonic signal was collected using a Mitutoyo $100 \mathrm{X}$ (NA $=0.7$, working distance $=6.0 \mathrm{~mm}$ ) objective. An interference filter was used to reject the first harmonic and allow only the second harmonic signal to reach the PMT (H6780; Hamamatsu Photonics K.K., Hamamatsu City, Japan). A fiber-lamp (used while not scanning to illuminate the sample through the collecting objective), pellicle-beam splitter (flipped into the beam path before the microscope objective when not scanning), lens, and CCD camera were used in order to image the sample and the focal spot of the laser.

We used the electro-optic modulator and quarter-wave plate combination to rotate the polarization direction of the linearly polarized laser light. An electro-optic modulator functions as a variable wave plate where the phase delay is proportional to the voltage applied across it. For linearly polarized input light, the electro-optic modulator produces elliptically polarized light. The quarter-wave plate that follows the electro-optic modulator converts the elliptically polarized light into linearly polarized light rotated by some angle with respect to the laser polarization direction. The degree of rotation is directly proportional to the applied voltage: from 0 degrees for $0 \mathrm{~V}$ to 180 degrees for the full-wave voltage of the electro-optic modulator. The voltage across the electro-optic modulator can be modulated at high frequencies; our system is limited to a bandwidth of $1 \mathrm{MHz}$.

A function generator (DS345; Stanford Research Systems, Sunnyvale, CA) provided a saw-tooth waveform at $4 \mathrm{kHz}$ that was amplified to have an amplitude equal to the full-wave voltage of the modulator using a high voltage power supply (Conoptics 302A). The polarization modulated beam produces a modulated SHG signal in the sample with frequency components at the first and second harmonic of the modulation frequency (the origin of these modulations is discussed in Theory, below) After passing through a current preamplifier (SR570; Stanford Research Systems, Sunnyvale, CA), the modulated signal from the PMT was decoded using three lock-in amplifiers (SR830; Stanford Research Systems., Sunnyvale, CA) set to detect the amplitude and phase of the SHG signal at both the first and second harmonic of the EOM modulation frequency as well as the optical chopper frequency. A Labview program was used to coordinate motion of the translation stage using a motion controller (ESP300; Newport, Irvine, CA) and Newport 850F actuators and to acquire data from the lock-in amplifiers.

\subsection{Sample preparation and modification}

\subsubsection{Tissue source}

Type I collagen fascicles were harvested from tails of 3-4 month old Sprague-Dawley rats that had been stored at -20 degrees C. We have previously observed that there is no difference between fresh and frozen tissue with respect to second harmonic signal (unpublished data). Individual tendon fascicles were removed from the tendon bundles under a dissecting microscope. Fascicles harvested from the rats ranged in length from $10 \mathrm{~cm}$ to $20 \mathrm{~cm}$, with a diameter ranging from 0.1 to $0.3 \mathrm{~mm}$. Some fascicles were structurally modified, as described below. Both control and modified samples were prepared for analysis as frozen sections; $4 \mu \mathrm{m}$ serial slices were obtained from approximately $20 \mu \mathrm{m}$ to $30 \mu \mathrm{m}$ below the surface. The unstained sections were mounted on glass slides beneath a cover slip.

\subsubsection{Non-enzymatic cross-linking}

Several agents were used for inducing covalent crosslinking in collagen: glutaraldehyde, formalin, methylglyoxal (MG), and glucose-6-phosphate (G-6-P). Glutaraldehyde and formaldehyde are potent chemical crosslinking agents used for tissue fixation; we selected them because they are capable of producing far higher levels of covalent bonds than occur naturally. G-6-P reacts with lysine residues on collagen nonenzymatically; over time these adducts undergo a series of complex reactions and rearrangements, culminating in the formation of advanced glycation-endproducts (AGEs). AGEs comprise a heterogeneous group of compounds,covalently bonded to collagen, that accumulate on collagen in virtually all tissues and organ systems, and which are associated with the profound structural and functional changes that occur in aging and diabetes ${ }^{19}$. G-6-P was selected as the glycating agent, as it reacts with collagen in vitro much more quickly 
than glucose. MG is a naturally occurring metabolic intermediate, which has recently been recognized as playing a role in AGE formation in vivo. In vitro studies have shown that incubation of collagen with MG results in rapid formation of AGE compounds. We selected this compound in order to generate collagen with a high level of AGE-type crosslinking.

Samples were placed in microcentrifuge vials to which were added one $\mathrm{ml}$ of one of the following solutions: $10 \%$ formalin, $5 \%$ glutaraldehyde in $500 \mathrm{mM}$ sodium phosphate buffer, $\mathrm{pH} \mathrm{7.1;100} \mathrm{mM} \mathrm{methylglyoxal} \mathrm{in} 250 \mathrm{mM}$ sodium phosphate buffer, $\mathrm{pH} 7.1$; $200 \mathrm{mM}$ G-6-P in $250 \mathrm{mM}$ sodium phosphate buffer, $\mathrm{pH}$ 7.1. All of the samples except for those incubated with G-6-P were incubated for $24 \mathrm{hrs}$ at room temperature. Samples in G-6-P were incubated for 1, 2, or 3 weeks. At the end of the incubation period, all samples were washed in phosphate buffered saline (PBS) and sectioned as described above.

\subsubsection{Thermal denaturation}

Rat tail tendon fascicles were placed in microcentrifuge vials to which was added $1 \mathrm{ml}$ of PBS at room temperature. The vials were placed for 45 minutes in a digital dry bath heater (Isotemp 125D; Fisher Scientific, Houston, TX), that had already reached its set temperature. The temperature display on the digital dry bath heater was calibrated against an $\mathrm{Hg}$ thermometer. The following temperatures (degrees $\mathrm{C}$ ) were used: 50.0, 51.0, 52.0, 52.5, 53.0, 53.5, 54.0, 55.0, 56.0, 56.5 , and 57.0. At the end of the heating period, the vials were removed from the heating block and allowed to cool to room temperature. Frozen sections were prepared as described above.

\subsubsection{Alcohol drying}

To determine the effect of collagen hydration on SHG, we compared normal fascicles with fascicles that had been subjected to dehydration through immersion in a graded series of ethanol dilutions, beginning with $25 \%$ ethanol in water, and ending in absolute ethanol. Samples were mounted in paraffin so that sections could be prepared.

\subsubsection{Collagenase digestion}

A solution of highly purified collagenase (Type VII collagenase; Sigma, Saint Louis, MO), containing 140 U/ml (1000$3000 \mathrm{CDU} / \mathrm{mg}$ ) in $0.01 \mathrm{M} \mathrm{CaCl}_{2} / 0.02 \mathrm{M}$ Tris $(\mathrm{pH} 7.55) / 0.05 \%$ toluene was prepared. Collagenase efficacy is determined by measuring liberation of leucine from the polypeptide after incubation for five hours in $0.01 \mathrm{M} \mathrm{CaCl}_{2} / 0.02$ $\mathrm{M}$ Tris at a pH of 7.4 and a temperature of 37 degrees $\mathrm{C}$. Efficacy is expressed in terms of Collagen Digestion Units (CDU); 1 unit (U) is equivalent to the liberation of $1.0 \mu \mathrm{M}$ of leucine. Rat tail tendon fascicles were incubated at 37 degrees $\mathrm{C}$ in this solution for $1 \mathrm{~h}, 2 \mathrm{~h}, 4 \mathrm{~h}, 6 \mathrm{~h}$, and $12 \mathrm{~h}$. Frozen sections were then prepared as discussed above.

\subsubsection{Age dependent cross-linking}

To examine the effects of age on SHG, we obtained a tail tendon sample from a 30 month old rat (available from a previous study) that had been stored at -20 degrees $C$. Frozen sections were prepared, and serial sections were obtained from $20 \mu \mathrm{m}$ to $30 \mu \mathrm{m}$ below the surface and mounted, unstained, on glass slides beneath a cover slip.

\section{$\underline{3.2 .7 \text { Control samples }}$}

Control samples were incubated at room temperature in PBS for $24 \mathrm{hrs}$.

\section{CAlibRATION}

In order to confirm the accuracy of the technique we use to measure the polarization dependence of SHG in collagen samples, we used the same techniques to make measurements in a quartz waveplate so that we could compare our experimental observations with the predictions of Eq. 6 . We performed twenty depth scans ( $1 \mu \mathrm{m}$ resolution) over a 100 $\mu \mathrm{m}$ axial range near the front surface of the quartz. The scans were spaced $1 \mu \mathrm{m}$ apart in the transverse direction. We obtained an average values of $R=0.26$, in good agreement with the theoretical value, $R=0.25$.

The quartz crystal also allows us to correct the measured value of $S$ for the fact that the chopper modulation is not a perfect sine wave (refer to Theory). In quartz, we expect $S_{c o r r}=1.33$ and we measure $S=0.93$. Thus, for any sample, the measured value of $S$ must be multiplied by 1.43 in order to obtain the correct ratio of the FMH signal to the DC signal in Eq. 3. 


\section{RESULTS}

\subsection{Overview}

Collagen is characterized not only by its well-defined structure - from the molecular to the macroscopic-but also by its continuous modification years after the initial structure has been assembled. We wanted to investigate the effect of some of these modifications on SHG signal under controlled conditions. To this end, we subjected isolated fascicles to conditions designed to produce perturbations in structural integrity analogous to those that occur in vivo. We examined the effects of increased nonenzymatic glycation, enzymatic digestion, and thermal denaturation. In addition, we examined the effects of inducing high levels of covalent crosslinking with agents such as glutaraldehyde and formaldehyde. We performed experiments to determine the effect of these changes on the polarization dependence of the SHG signal. We decided to focus on polarization dependence because this measurement has a much smaller intrinsic variability than the intensity, which tends to fluctuate over several orders of magnitude even within highly organized samples such as rat tail tendon.

\subsection{Non-enzymatic cross-linking}

$R$ was measured in sections from rat tail tendon tissue crosslinked with methylglyoxal (MG), G-6-P, glutaraldehyde or formaldehyde. For each of the samples, we randomly chose three regions to scan. However, we tried to avoid regions where the section was folded over on itself, crumpled, or torn; our previous results "indicate that such phenomena can have a significant effect on $R$. Each region consisted of a $150 \mu \mathrm{m}$ by $100 \mu \mathrm{m}$ rectangle in the plane of the frozen section. Data were collected with a scan resolution of $2 \mu \mathrm{m}$ ( 15,000 points per region). Data points where the signal at the first modulation harmonic did not exceed a minimum threshold (about ten times the noise floor) were eliminated. For each of the three scans obtained in each sample, the average value of $R$ was determined. The corresponding value of $\frac{d_{X Y Y}}{d_{X X X}}-$ the ratio of the two independent elements in the collagen second order nonlinear susceptibility tensor-was calculated and plotted in Figure 3. There were no significant differences between control collagen and any of the crosslinked collagen with respect to $\frac{d_{X Y Y}}{d_{X X X}}$. We also measured $\frac{d_{X Y Y}}{d_{X X X}}$ in collagen obtained from an old rat, to determine if AGE accumulation that had occurred naturally over an extended period of time might differ in some way from crosslinking induced artificially at an accelerated rate. However, $\frac{d_{X Y Y}}{d_{X X X}}$ in aged tissue was not significantly different from that of control tissue (Figure 3).

\subsection{Thermal denaturation}

We measured SHG in collagen that had been heated to different temperatures for 45 minutes. Samples subjected to temperatures less than $\sim 52$ degrees $C$ had a normal SHG signal; samples heated to a temperature exceeding $\sim 54$ degrees $C$ had no SHG signal. Some samples heated to $\sim 53.0$ degrees $C$ had no signal, while others had normal signal. Samples had either a normal SHG signal or no signal at all, suggesting a sharp transition around 53 degrees. For each sample, we determined the average value of $R$ in three randomly selected $100 \mu \mathrm{m}$ by $100 \mu \mathrm{m}$ regions $(1 \mu \mathrm{m}$ scan resolution). We again calculated the corresponding value of $\frac{d_{X Y Y}}{d_{X X X}}$. The effects of heat on $\frac{d_{X Y Y}}{d_{X X X}}$ (in those samples in which signal was detectable) is shown in Figure 4. There is no significant difference (with respect to $\frac{d_{X Y Y}}{d_{X X X}}$ ) between the heated samples in which signal was detected and the control samples.

\subsection{Collagenase digestion}

We measured SHG signal in collagen digested with collagenase at 37 degrees $\mathrm{C}$ for different time periods. Tissue treated for $4 \mathrm{~h}$ had normal signal; no signal was detectable in any tissue treated for $6 \mathrm{~h}$ or longer. For each sample, we 
determined the average value of $R$ in three randomly selected $50 \mu \mathrm{m}$ by $50 \mu \mathrm{m}$ regions $(1 \mu \mathrm{m}$ scan resolution). The effects of collagenase digestion on the calculated values of $\frac{d_{X Y Y}}{d_{X X X}}$ are shown in Figure 5. There is no significant difference (with respect to $\frac{d_{X Y Y}}{d_{X X X}}$ ) between the collagenase digested samples in which signal was detected and the control samples.

\subsection{Hydration state}

In a previous study, we had observed a significant change in $R$ between native rat tail tendon samples stored in PBS and allowed to air-dry and tendons that were dehydrated in a series of increasingly concentrated ethanol baths ${ }^{11}$. However, since the studies were performed in native rat tail tendon, we were unable to determine conclusively whether the observed change in $R$ was attributable to changes in the nonlinear susceptibility or to changes in the linear optical properties. In the present study, we examined the effect of hydration on SHG in thin sections of rat tail tendon in order to minimize confounding effects of changes in the linear optical properties. We measured $R$ (and calculated the corresponding value of $\frac{d_{X Y Y}}{d_{X X X}}$ ) in six randomly selected $100 \mu \mathrm{m}$ by $100 \mu \mathrm{m}$ regions ( $1 \mu \mathrm{m}$ scan resolution). We found that the value of $\frac{d_{X Y Y}}{d_{X X X}}$ in the dehydrated sections was lower than that in the control sections (Figure 6).

\section{$5.6 R, S$, and $\gamma$}

In the results presented above, we have focused on measuring $R$-the ratio between the second harmonic signal modulated at double the electro-optic modulator frequency and the signal modulated at the electro-optic modulator frequency. From a measured value of $R$, we can directly calculate $\gamma$, a parameter of the second order nonlinear susceptibility tensor of collagen. However, as discussed in Theory, we can also measure the ratio $S$ between the signal at the electro-optic modulator frequency and the signal at the chopper frequency. We noted previously (see Calibration) that the measured value of $S$ must be corrected in order to take into account the fact that the chopper modulation is not a sine wave; we use measurements in quartz to obtain this correction factor. Once $S$ has been multiplied by the correction factor, it can also be used to directly calculate $\gamma$. We wanted to determine if the values of $\gamma$ obtained from measurements of $R$ and $S$ agreed. To do so, we measured both $R$ and $S$ in the same 100 by $100 \mu \mathrm{m}$ region of a sample using a $1 \mu \mathrm{m}$ scan resolution; we obtained mean values of $R=1.30$ and $S=0.15$. We multiply $S$ by the correction factor (1.43) to obtain $S_{\text {corr }}=0.21$. We can then calculate $\gamma$ from $R$ to obtain $\gamma=-0.60$, and we can calculate $\gamma$ from $S$ to also obtain $\gamma=-$ 0.60. The good agreement between these two results offers additional support for the cylindrical symmetry model of SHG in collagen. Finally, given $\gamma$, we can calculate the ratio of the two independent elements in the second order nonlinear susceptibility tensor for this example: $\frac{d_{X Y Y}}{d_{X X X}}=0.75$. This ratio ranges between about 0.63 and 0.75 in all of our control samples; $\frac{d_{X Y Y}}{d_{X X X}}=0.69$ is an average value.

\section{DISCUSSION}

We have measured the effect of several biologically relevant modifications of collagen structure-non-enzymatic crosslinking, thermal denaturation, collagenase digestion, and dehydration-on the polarization dependence of SHG. We found that non-enzymatic cross-linking had no observable effect on the polarization dependence-whether samples were crosslinked in vitro or whether crosslinking occurred naturally through aging. These findings were surprising, since alterations in crosslinking are known to have significant effects on many aspects of collagen structure. For example, glycation has been shown to significantly increase molecular packing ${ }^{22}$. Similarly, collagen that has been subjected to 
strong chemical crosslinking agents such as glutaraldehdye and formaldehyde has extensive alterations in its structure at both the molecular and supramolecular level $\mathrm{l}^{23.24}$. Virtually the only aspect of collagen that remains unchanged by such treatment is its primary structure.

Thermal denaturation resulted in an abrupt drop in the SHG signal, but we did not observe that the polarization dependence of the signal changed in samples heated to just below the point of denaturation. Changes that did not result in destruction of the fibrillar structure did not result in a change in the SHG signal. A previous study of SHG in thermally denatured collagen-using purified type I collagen fibrils, a much larger sample, and a much larger focal spot $(\sim 0.5 \mathrm{~cm})$-found that SHG signal became undetectable at a temperature between 65 degrees $\mathrm{C}$ and 70 degrees $\mathrm{C}^{25}$. This study also found that for temperatures of 30 degrees $C$ to 50 degrees $C$, the SHG signal dropped at the same rate as the fluorescence signal; at higher temperatures, the SHG signal dropped more rapidly. The larger samples and the use of purified collagen may account for the fact that SHG signal was observed at temperatures up to 65 degrees C, a temperature at which our samples were completely denatured. The temperature at which Theodossiou et. al. ${ }^{25}$ observed an increase in the rate of SHG decay corresponds to the temperature ( 23 degrees $C)$ at which we noticed complete denaturation of the tissue.

In the collagenase-digested samples, we obtained similar results as in the case of thermal denaturation. Samples that were digested for $2 \mathrm{hr}$ or $4 \mathrm{hr}$ did not exhibit a SHG polarization dependence that was significantly different from the control samples. A sample that was digested for $6 \mathrm{hr}$ - where the fibrillar structure was destroyed—did not have any SHG signal.

We observed that significant changes in the degree of hydration do have an effect on the polarization dependence of the second harmonic signal. The value of $\frac{d_{X Y Y}}{d_{X X X}}$ measured in dry tissue is lower than that in hydrated tissue. Based on measurements in thick samples-where birefringence and polarization dependent scattering may also play a role-we had postulated that the absence of highly polar water molecules reduces the nonlinear polarizability normal to the fibril axis, which would result in a decreased value of $\frac{d_{X Y Y}}{d_{X X X}} "$. Our results in thin sections, where birefringence and polarization dependent scattering can be neglected, support our hypothesis.

\section{CONCLUSION}

The effects of structural perturbations on SHG in collagen were assessed in this study. We chose to investigate polarization dependence of the SHG signal because it fluctuates much less than does the magnitude of the signal. Not surprisingly, we observed that when collagenase and thermal denaturation destroy the fibrillar structure of collagen, the second harmonic signal is lost completely. We found that the hydration state of collagen significantly affected the polarization dependence of SHG; we hypothesize that the absence of highly polarizable water molecules may be responsible for this change. The most surprising finding was the complete absence of any change in SHG polarization dependence in collagen that had been subjected to the most extensive structural modification-short of denaturation-in our study. This observation raises interesting questions about the structural aspects of collagen most significantly involved in determining the second order nonlinear susceptibility. Clearly, experiments targeting other structural features, such as degree of chirality or perhaps even primary structure itself may shed light on this fascinating problem.

\section{ACKNOWLEDGEMENTS}

This work was supported by grants from the National Institutes of Health, 1 R01 EB 00337-02 and from the Center of Excellence for Laser Applications in Medicine, U.S. Department of Energy DE-FG03-98ER62576. This work was performed under the auspices of the U.S. Department of Energy at Lawrence Livermore National Laboratory under contract W-7405-ENG-48. 


\section{REFERENCES}

1. P. J. Campagnola, H. A. Clark, W. A. Mohler, A. Lewis, and L. M. Loew. "Second-harmonic imaging microscopy of living cells." Journal of Biomedical Optics. 6:277-286. 2001.

2. P. J. Campagnola. A. C. Millard, M. Terasaki, P. E. Hoppe, C. J. Malone, and W. A. Mohler. "Three-dimensional high-resolution second-harmonic generation imaging of endogenous structural proteins in biological tissues." Biophysical Journal. 81:493-508. 2002.

3. P.-C. Cheng, C.-K. Sun, B. L. Lin, F.-J. Kao, S.-W. Chu. "Biological multi-modality nonlinear spectromicroscopy: multiphoton fluorescence, second-and third-harmonic generation." Proceedings of Scanning. 23:109-1 10. 2001.

4. Y. Guo, P. P. Ho, A. Tirksliunas, F. Lui, R. R. Alfano. "Optical harmonic generation from animal tissues by the use of ps and fs laser pulses." Applied Optics. 35:6810-6813. 1996.

5. Y. Guo, P. P. Ho, H. Savage, D. Harris, P. Sacks, S. Schantz, F. Liu, N. Zhadin, and R. R. Alfano. "Second-harmonic tomography of tissue." Optics Letters. 22:1323-1325. 1997.

6. B-M Kim, J. Eichler, and L. B. Da Silva. "Frequency doubling of ultrashort laser pulses in biological tissues." Applied Optics. 38:7145-7150. 1999.

7. B-M Kim, J. Eichler, K. M. Reiser, A. M. Rubenchik, L. B. Da Silva. "Collagen structure and nonlinear susceptibility: effect of heat, glycation, and enzymatic cleavage on second harmonic signal intensity." Lasers in Surgery and Medicine. 27:329-335. 2000.

8. K. König. "Multiphoton microscopy in life sciences." Journal of Microscopy. 200:83-104. 2000.

9. J. Squier and M. Müller. "High resolution nonlinear microscopy: a review of sources and methods for achieving optimal imaging." Review of Scientific Instruments. 72:2855-2867. 2001.

10. P. Stoller, B-M. Kim, K.M. Reiser, A. M. Rubenchik, and L. B. Da Silva. "Polarization dependent optical second harmonic imaging of rat-tail tendon." Journal of Biomedical Optics. 7:205-214. 2002 a.

11. P. Stoller, K. M. Reiser, P. M. Celliers, A. M. Rubenchik. "Polarization-modulated second harmonic generation in collagen." Biophysical Journal. 82:3330-3342. 2002 b.

12. R. M. Williams, W. R. Zipfel, and W. W. Webb. "Multiphoton microscopy in biological research." Current Opinion in Chemical Biology: 5:603-608. 2001.

13. A. Zoumi, A. Yeh, and B. J. Tromberg. "Imaging cells and extracellular matrix in vivo by using second-harmonic generation and two-photon excited fluorescence." Proc. of the Natl. Acad. of Sciences. 99:11014-11019. 2002.

14. K. E. Kadler, D. F. Holmes, J. A. Trotter, and J. A. Chapman. "Collagen fibril formation." Biochem. J. 316:1-11. 1996.

15. L. Knott and A. J. Bailey. "Collagen cross-links in mineralizing tissues: a review of their chemistry, function and clinical relevance." Bone. 22:181-187. 1998.

16. K. M. Reiser, R. J. McCormick, R. B. Rucker. "Enzymatic and nonenzymatic cross-linking of collagen and elastin." FASEB J. 6:2439-2449. 1992.

17. K. M. Reiser. "Nonenzymatic glycation of collagen in aging and diabetes." Proceedings of the Society of Experimental Biology" and Medicine. 196:17-29. 1991.

18. K. M. Reiser. "Extracellular matrix." Encyclopedia of Gerontology. 1:519-529. 1996.

19. K. M. Reiser. "Nonenzymatic glycation of collagen in aging and diabetes." Proceedings of the Society of Experimental Biology and Medicine. 218:23-37. 1998.

20. I. Freund, M. Deutsch, and A. Sprecher. "Connective tissue polarity: Optical second-harmonic microscopy, crossedbeam summation, and small-angle scattering in rat tail tendon." Biophysical Journal. 50:693-712. 1986.

21. R.W. Boyd. Nonlinear Optics. Academic Press, San Diego. 1992.

22. S. Tanaka, G. Avigad, B. Brodsky, and E. F. Eikenberry. "Glycation induces expansion of the molecular packing of collagen," Journal of Molecular Biology 203:495-505. 1988.

23. M. E. Nimni, D. T. Cheung, B. Strates, M. Kodama, and K. Sheikh. "Bioprosthesis derived from cross-linked and chemically modified collagenous tissues." In Collagen: Biotechnology. Marcel E. Nimni, editor. Volume 3. pp. 1-38. CRC Press, Boca Raton. 1988.

24. E. Heidemann. "The chemistry of tanning." In Collagen: Biotechnology. Volume 3. Marcel E. Nimni, editor. Volume 3. pp. 39-61. CRC Press, Boca Raton. 1988.

25. T. Theodossiou, G. S. Rapti, V. Hovhannisyan, E. Georgiou, K. Politopoulos, and D. Yova. 2002. "Thermally induced irreversible conformational changes probed by optical second harmonic generation and laser-induced thuorescence," Lasers in Med. Sci. 17:34-41. 


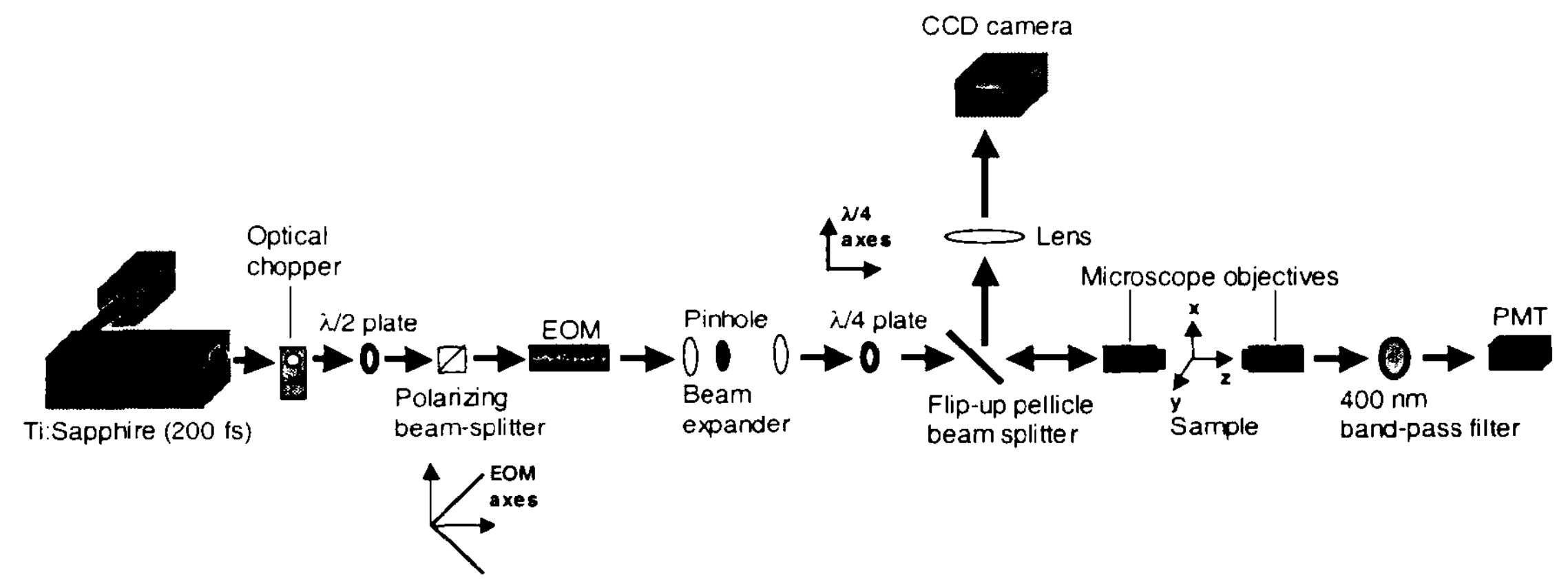

Figure 1. Diagram of the experimental setup.

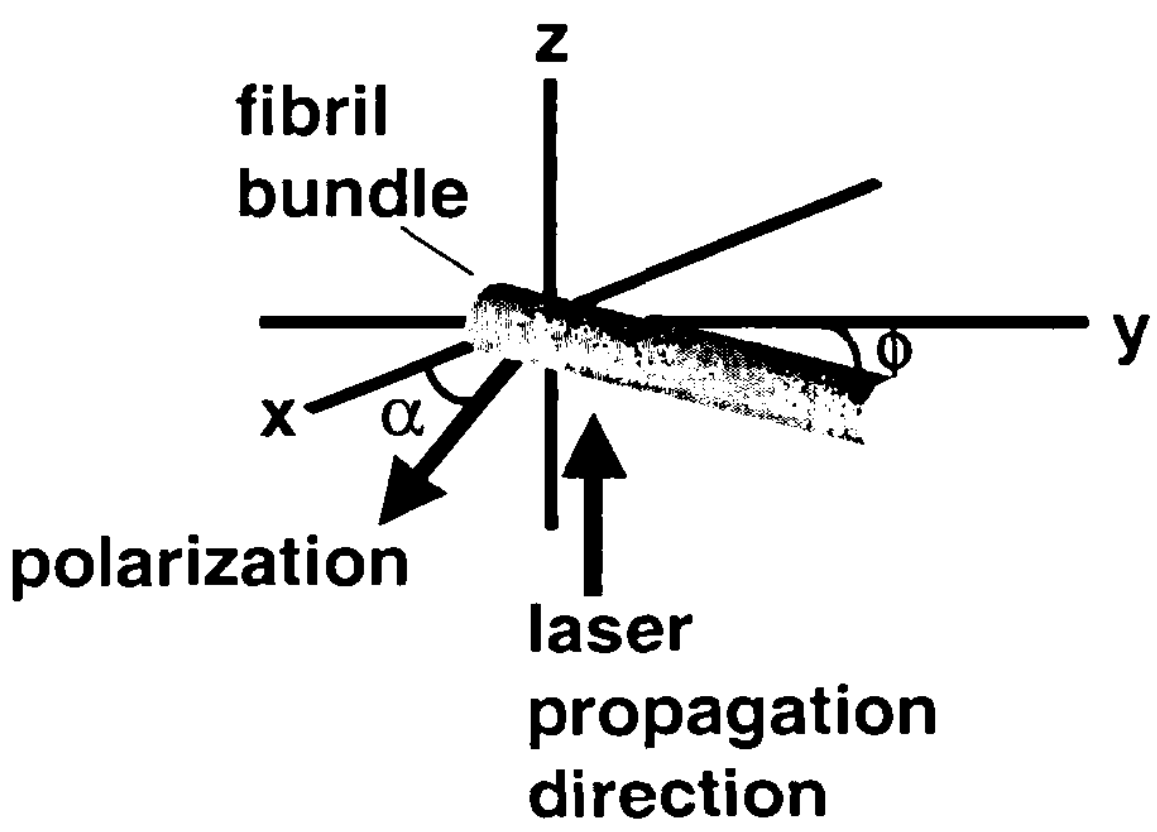

Figure 2. Sketch of the fiber orientation and laser beam propagation direction and polarization.

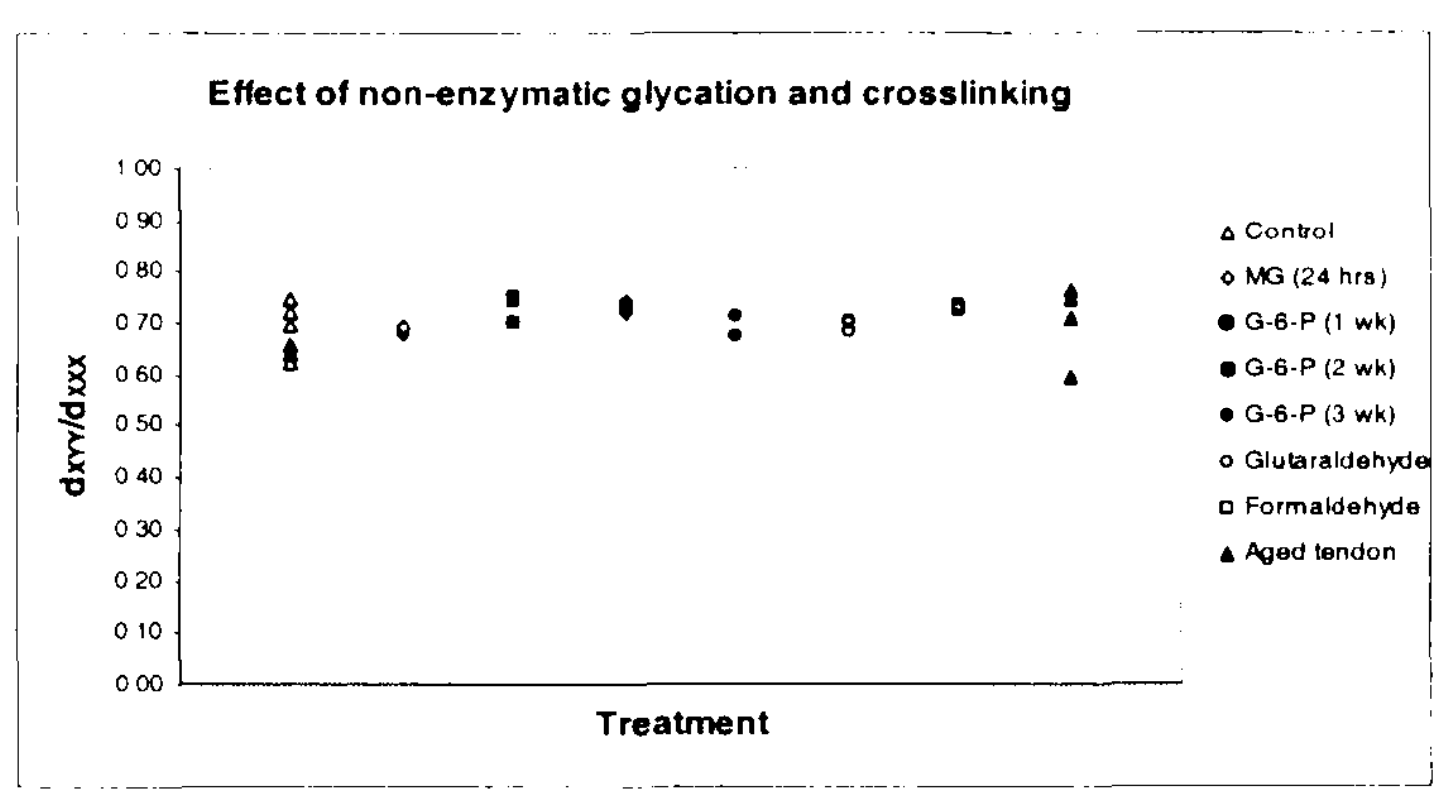

Figure 3. Scatter-plot of the effect on $\frac{d_{X Y Y}}{d_{X X X}}$ of non-enzymatic cross-linking. The mean value measured for each region scanned is plotted as a single point. 


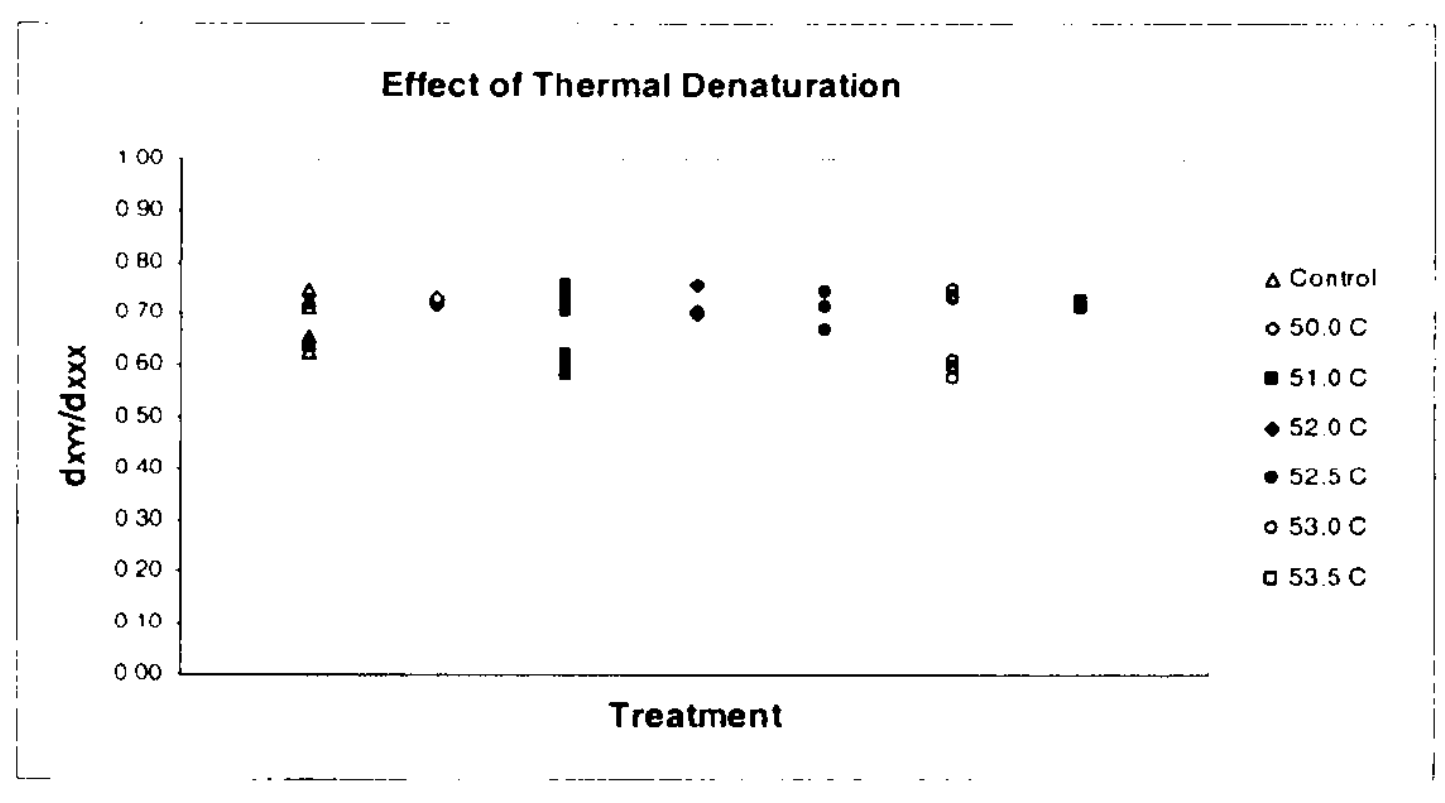

Figure 4. Scatter-plot of the effect on $\frac{d_{X Y Y}}{d_{X X X}}$ of heating the samples. The mean value measured for each region scanned is plotted as a single point. Note that two different samples were heated to 51.0 degrees $C$ and 53.0 degrees $C$; samples heated to 54.0 degrees C. 55.0 degrees C. 56.0 degrees C. 56.5 degrees $C$. and 57.0 degrees $C$ had no detectable signal.

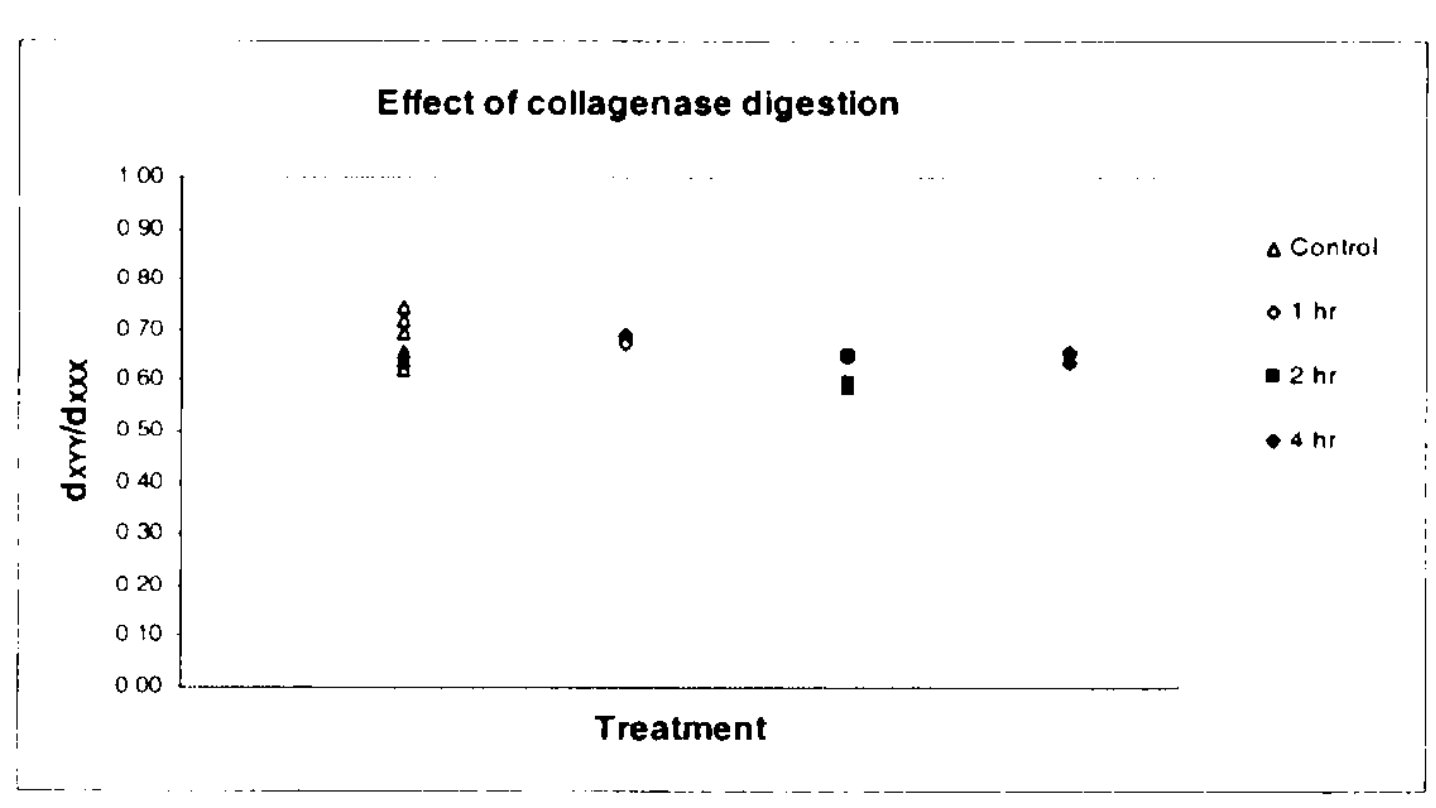

Figure 5. Scatter-plot of the effect on $\frac{d_{X Y Y}}{d_{X X X}}$ of collagenase. The mean value measured for each region scanned is plotted as a single point. Samples incubated with collagenase for $6 \mathrm{hr}, 12 \mathrm{hr}$, and $24 \mathrm{hr}$ had no detectable signal.

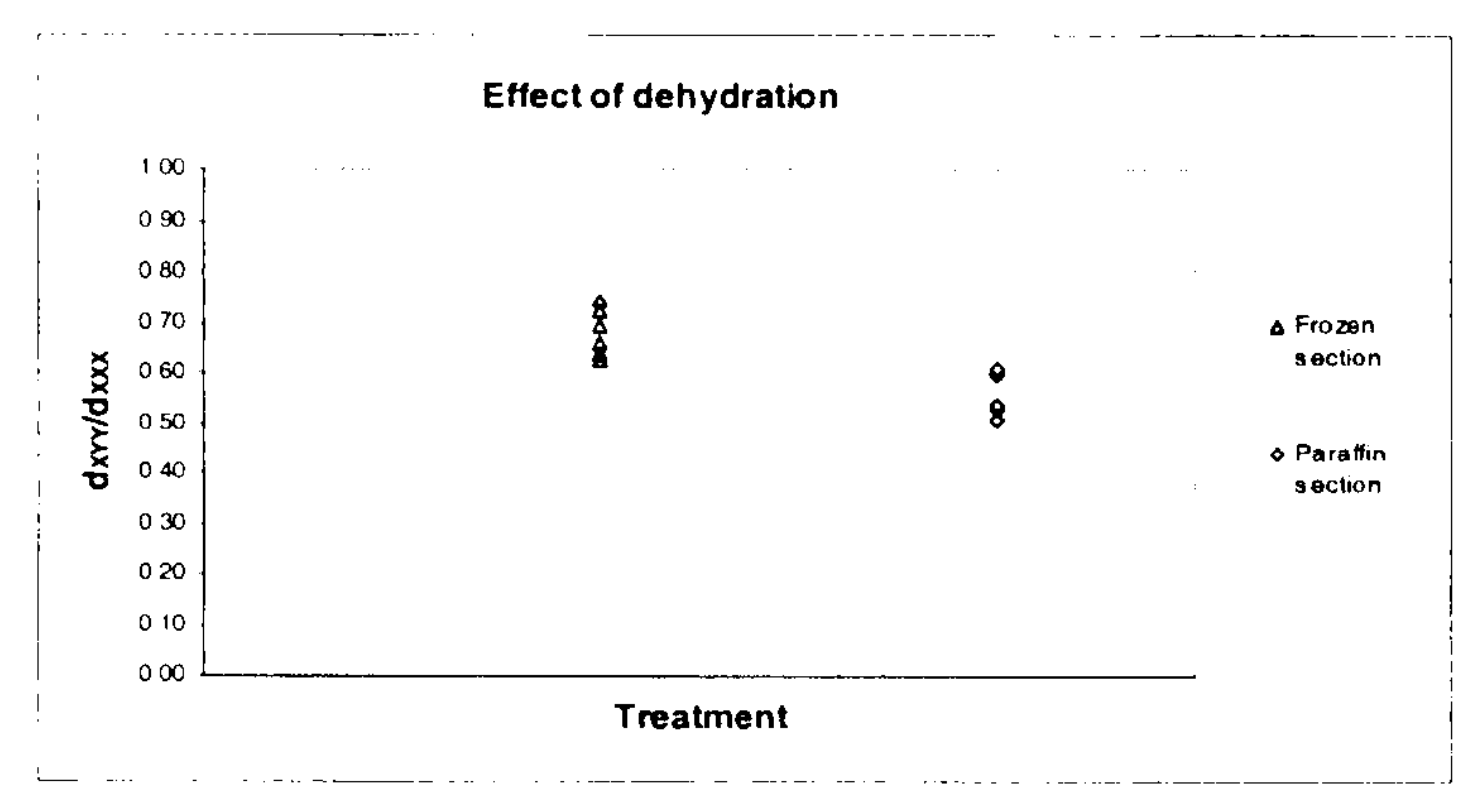

Figure 6. Scatter-plot of the effect on $\frac{d_{X Y Y}}{d_{X X X}}$ of hydration. The mean value measured for each region scanned is plotted as a single point. 